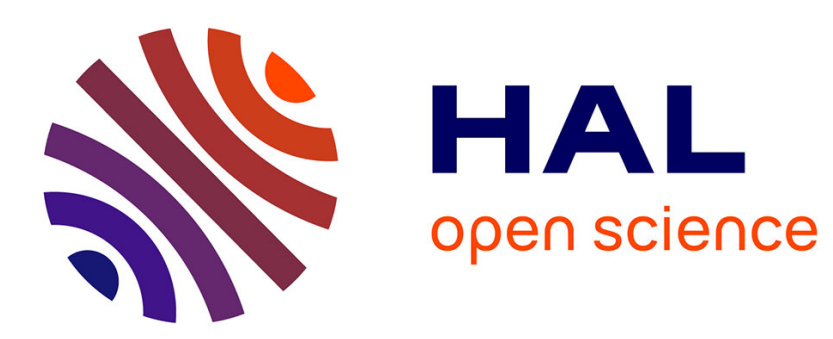

\title{
L'impact des chocs externes dans les économies du Mercosur: un modèle VAR Structurel Céline Gimet
}

\section{To cite this version:}

Céline Gimet. L'impact des chocs externes dans les économies du Mercosur: un modèle VAR Structurel. Économie Internationale, 2007, 110, pp. 107-136. halshs-00356105

\section{HAL Id: halshs-00356105 \\ https://shs.hal.science/halshs-00356105}

Submitted on 26 Mar 2009

HAL is a multi-disciplinary open access archive for the deposit and dissemination of scientific research documents, whether they are published or not. The documents may come from teaching and research institutions in France or abroad, or from public or private research centers.
L'archive ouverte pluridisciplinaire HAL, est destinée au dépôt et à la diffusion de documents scientifiques de niveau recherche, publiés ou non, émanant des établissements d'enseignement et de recherche français ou étrangers, des laboratoires publics ou privés. 


\title{
L'IMPACT DES CHOCS EXTERNES DANS LES ECONOMIES DU MERCOSUR : UN MODELE VAR STRUCTUREL
}

\author{
Céline Gimet ${ }^{*}$
}

RESUME. Cette étude vise à déterminer les causes de la déstabilisation des pays du Mercosur ces dernières années, à l'origine du flottement des monnaies. A l'aide d'un VAR Structurel, nous analysons l'impact d'une politique monétaire restrictive des Etats-Unis, d'une baisse de la confiance des marchés financiers, et d'une hausse des prix mondiaux des produits agricoles sur les secteurs réel, financier et monétaire de ces pays. La vulnérabilité des économies à ces chocs révèle le défaut de soutenabilité des régimes de change en place durant cette période. En outre, bien que les réactions des pays à chaque type de choc soient proches, des divergences subsistent du fait de l'hétérogénéité des structures macroéconomiques et financières des pays.

Classification JEL: C32, E42, F41

Mots-clefs : Chocs Internationaux, Mercosur, modèle Var Structurel, restrictions contemporaines et de long terme.

\begin{abstract}
The paper deals with the reasons heaving led the Mercosur countries to let their currency float at the end of the nineties. By the means of a Structural VAR model, we analyse the impact of a restrictive monetary policy in the United States, a decrease in the financial markets confidence and an increase of the agricultural raw materials prices in the real, financial and monetary sectors of these countries. Their vulnerability to these shocks reveals the lack of sustainability of the exchange rate regimes during that period. Besides, although the reactions of the countries are close, some divergences remain because of the macroeconomic and financial structural heterogeneity of these economies.
\end{abstract}

JEL Classification: C32, E42, F41

Keywords: International Shocks, Mercosur, Structural Var Model, Short Term and Long Term Restrictions.

\footnotetext{
"Centre d'Economie et de Finances Internationales (CEFI).Université de la Méditerranée. Château Lafarge, Route des Milles 13290 Les Milles, Aix-en-Provence. E-mail : celine.gimet@free.fr.

Je tiens à remercier les deux rapporteurs anonymes de la Revue pour leurs remarques constructives.
} 


\section{INTRODUCTION}

Au début de la décennie quatre-vingt-dix, les pays du Mercosur et leurs membres associés avaient opté pour des solutions de change plutôt fixes. Or, les pays ont connu d'importantes crises financières qui se sont succédé jusqu'en 2001. Ainsi, au lendemain de la crise argentine, seule la Bolivie a conservé un système de crawling peg, les autres pays de la zone ayant été contraints de laisser flotter leur monnaie. II importe donc de s'interroger sur les facteurs à l'origine de la déstabilisation de ces pays.

De nombreuses études empiriques ont mis l'accent sur la vulnérabilité des économies émergentes à des chocs externes. Plusieurs instruments statistiques ont été utilisés à cette fin, notamment des modèles vectoriels autorégressifs (VAR), (Calvo et Mendoza, 1998 ; Bordo et Murshid, 2002). Cependant, la méthode Vectorielle Autorégressive Structurelle (SVAR) est plus précise, car elle permet d'imposer certaines hypothèses concernant les réactions des variables à différents types de chocs, en référence à la théorie économique, ce qui permet une meilleure interprétation des résultats. Les auteurs qui ont appliqué cette méthode se sont intéressés, en particulier, aux conséquences d'un changement de la politique monétaire des Etats-Unis sur les pays et en ont déduit que les réactions des économies émergentes (Canova, 2003; Mackowiak, 2006) diffèrent de celles observées dans les pays industrialisés (Cushman et Zha, 1997; Kim et Roubini, 2000). En effet, les pays émergents, très sensibles à ce type de choc du fait de leur dépendance vis-à-vis des conditions économiques externes, répondent généralement à une politique monétaire américaine restrictive par une forte augmentation de leur taux d'intérêt, ce qui provoque une contraction de leur production. En outre, cette méthode offre la possibilité de refléter le degré de symétrie des chocs entre plusieurs pays, et de comparer la vitesse avec laquelle ces économies s'ajustent à ces chocs. C'est le niveau de convergence des structures macroéconomiques et financières qui explique le caractère asymétrique des réponses des pays à un même choc. Ainsi, au sein d'une région, il est possible, au terme de l'analyse, d'identifier un noyau de pays présentant un profil économique et financier proche, et ayant par conséquent des réactions à des chocs communs identiques, grâce à l'étude de la corrélation des chocs ou des réponses aux chocs entre les pays de la zone (Bayoumi et Eichengreen, 1993 ; Canova, 2003).

Dans notre étude, nous avons élargi le champ d'analyse en prenant en compte de nouveaux chocs externes. En effet, les phénomènes de contagion qui sont apparus à la suite des crises récentes soulignent la nécessité de considérer l'influence des crises financières dans d'autres pays émergents (Kaminsky, Reinhart et Vegh, 2003). En outre, les économies du Mercosur, principalement exportateurs de produits agricoles, sont sensibles aux fluctuations du prix de ces biens sur les marchés mondiaux. La réaction de la production des pays, notamment du Brésil, de l'Uruguay et du Chili, à la suite de l'accroissement de la demande de la Chine ces dernières années illustre bien cette situation.

Nos résultats montrent que, quel que soit le régime de change en place, les pays réagissent largement à des variations externes, notamment sur les marchés boursiers. En outre, les répercussions néfastes de ces chocs se transmettent de la sphère financière dans le domaine réel avec pour conséquence un ralentissement important de la production. Ce phénomène met en évidence le manque de soutenabilité des régimes de change en place durant la dernière décennie, ce qui 
explique l'importante sensibilité des pays aux variations externes. C'est pourquoi les pays ont subi largement les conséquences négatives des crises financières qui ont touché les pays émergents.

Notre étude se divise en trois parties. La première section présente le modèle VAR Structurel sur lequel repose notre étude. Nous préciserons alors les restrictions de court et de long terme que nous avons retenues en référence à la littérature théorique. La seconde section décrit les résultats de notre analyse. Enfin, la troisième section étudie les similitudes qui existent entre les pays grâce à un test de corrélation des réponses des pays à chaque type de chocs.

\section{SPECIFICATION DU MODELE VAR STRUCTUREL}

\section{La modélisation VAR Structurelle}

Soit la représentation vectorielle autorégressive $\operatorname{VAR}(q)$ du modèle sous forme réduite :

$$
A(L) \Delta Y_{t}=e_{t}
$$

où $q$ est le nombre de retards, $Y_{t}$ est le vecteur des variables observables de dimension $n \times 1$, avec $n$ le nombre de variables du modèle et $e_{t}$ est un bruit blanc.

En vue d'obtenir les fonctions de réponse aux chocs ainsi que les décompositions de la variance de l'erreur de prévision, il est nécessaire d'écrire ce processus sous la forme moyenne mobile infinie structurelle. Pour ce faire, une étape intermédiaire consiste à « inverser » le modèle VAR canonique selon le théorème de Wold pour obtenir le VAR canonique sous forme moyenne mobile :

$$
\Delta Y_{t}=C(L) e_{t}
$$

avec $C(0)=I_{n}$ et $e_{t}$ le vecteur des innovations canoniques.

D'où la forme moyenne mobile structurelle du VAR :

$$
\Delta Y_{t}=\Theta(L) \varepsilon_{t}
$$

avec

$$
e_{t}=P \varepsilon_{t}
$$

où $P$ est une matrice de passage inversible $n \times n$ qu'il faut estimer en vue d'identifier les chocs structurels. Les contraintes de court terme se traduisent par la nullité de certains coefficients de la matrice $P$. La matrice $\Theta_{j}$ représente les fonctions de réponse aux chocs $\varepsilon_{t}$ des éléments de $\Delta Y_{t}$. On suppose que les différents chocs structurels ne sont pas corrélés entre eux et ont une variance unitaire ${ }^{2}$ :

$$
E\left(\varepsilon_{t}, \varepsilon_{t}^{T}\right)=I_{n}
$$

\footnotetext{
${ }^{2}$ L'exposant $T$ désigne la transposée de la variable concernée.
} 
$\Omega$ étant la matrice des variances covariances des innovations canoniques $e_{t}$, on a :

$$
E\left(e_{t}, e_{t}^{T}\right)=P E\left(\varepsilon_{t}, \varepsilon_{t}^{T}\right) P^{T}=P P^{T}=\Omega
$$

L'absence de réponse dans le long terme d'un certain nombre de variables $\Delta Y_{t}$ aux chocs $\varepsilon$ se traduit par la nullité du multiplicateur de long terme dynamique correspondant $^{3}$.

\section{Le choix des variables}

Nous travaillons sur quatre pays du Mercosur et leurs plus anciens membres associés: Argentine, Brésil, Bolivie, Chili, Paraguay, Uruguay, sur la période 1991M1, 2004M3, en périodicité mensuelle. L'objectif est d'étudier les conséquences des chocs internationaux dans les secteurs réel, financier et monétaire de chaque pays, ainsi que des interactions entre les différentes sphères de l'économie. Cinq variables domestiques et trois chocs externes ont été sélectionnés.

Dans notre modèle, chaque économie du Mercosur est décrite par le vecteur des variables endogènes $\Delta Y=\left(\begin{array}{c}\Delta e x t \\ \Delta y \\ \Delta r e r t e \\ \Delta a s s e t \\ \Delta m \\ \Delta \text { ninrte }\end{array}\right)$ où ext est la variable externe ${ }^{4}, y$ est la production, rerte le taux de change réel, asset la part des actifs étrangers détenus par la Banque Centrale, $m$ la masse monétaire M2 et ninrte le taux d'intérêt nominal correspondant au taux du marché monétaire de court terme. L'ordre des variables est choisi afin de faciliter la mise en place de contraintes de court et de long terme. II s'agit des variables traditionnellement utilisées dans la littérature concernant les VAR Structurels dès lors que l'objectif est de prendre en compte à la fois la dimension réelle et monétaire de l'économie aussi bien sous l'angle de l'offre que de la demande. Les deux premières et les deux dernières variables domestiques de notre modèle renvoient à la littérature traitant de l'impact des chocs monétaires sur les cycles économiques avec plus récemment la mise en évidence de l'importance du taux de change réel en tant que mécanisme de transmission des chocs. La décomposition entre les chocs d'offre et de demande, monétaires et réels s'appuie sur la même logique que celle de Gali (1992), Clarida et Gali (1994) et Sims et Zha (1999). Par ailleurs, le rôle des fluctuations internationales a été souligné en particulier par Cushman et Zha (1997), Kim et Roubini (2000), Canova (2003), Mackowiak (2006). Enfin, la nécessité de considérer les mouvements de capitaux internationaux dans les économies émergentes traduisant dans une certaine mesure la réaction des préteurs internationaux à la suite d'un choc externe, et ayant des conséquences néfastes sur le taux de change réel, a été révélée plus récemment

\footnotetext{
${ }^{3}$ Le détail des calculs est reporté dans l'annexe 1.

${ }^{4}$ ext représente la variable internationale, extérieure à l'économie du pays étudié, dont la définition est donnée plus loin.
} 
par la littérature sur les crises de change de troisième génération mais avec pour fondement le modèle VAR de Calvo, Leiderman et Reinhart (1994).

Par conséquent, le vecteur des chocs structurels associé à chaque variable prend la forme suivante $\varepsilon_{t}=\left(\begin{array}{c}\varepsilon_{e x t} \\ \varepsilon_{s} \\ \varepsilon_{d} \\ \varepsilon_{f i} \\ \varepsilon_{m d} \\ \varepsilon_{m s}\end{array}\right)$ où $\varepsilon_{e x t}, \varepsilon_{s}, \varepsilon_{d}, \varepsilon_{f i}, \varepsilon_{m d}, \varepsilon_{m s}$ représentent respectivement le choc externe, le choc d'offre réel domestique, le choc de demande réel domestique, le choc financier domestique, le choc de demande monétaire domestique, le choc d'offre monétaire domestique.

Les perturbations externes retenues sont un choc positif du taux d'intérêt des EtatsUnis (usintrte), en prenant en compte le taux d'intérêt monétaire de court terme du pays, du prix des produits agricoles (wprices), et de la variation de l'indice boursier composite des pays émergents proposé par Standard and Poor's ${ }^{5}$ (emei). La volatilité de cet indicateur a été calculée à partir d'un modèle GARCH qui donne la variance conditionnelle de cet indice à partir de laquelle l'écart type peut être déduit ${ }^{6}$. Le choc positif du taux d'intérêt aux Etats-Unis (usintrte) peut être considéré comme une politique monétaire restrictive du pays dans la mesure où il est associé à une augmentation du taux d'intérêt et qu'elle engendre une diminution de la masse monétaire, une baisse de la production et du niveau des prix dans l'économie (Sims, 1992 ; Sims et Zha,1999) ${ }^{7}$.Les résultats anticipés concernant les effets d'une politique monétaire restrictive des Etats-Unis sur les économies émergentes dépendent du degré d'intégration financière des pays avec les Etats-Unis et varient selon le régime de change en place. En théorie, les pays ayant un taux de change flottant sont moins exposés. En effet, les pays dont le taux de change est fixe connaissent des variations de taux d'intérêt plus fortes alors que l'inflation reste limitée. En revanche, en change flottant, l'augmentation du taux d'intérêt domestique reste faible mais l'inflation augmente de manière significative.

Par ailleurs, lorsque les pays ont un avantage comparatif dans le secteur agricole, ils sont particulièrement sensibles à une fluctuation du prix de ces biens. Une augmentation de ce prix engendre une augmentation des revenus et donc de l'emploi dans les secteurs concernés. Par l'effet du multiplicateur, cette situation se propage au niveau de l'économie entière. Dans le très court terme, les importations restent inchangées, il s'en suit alors une entrée de devises qui contribue à une expansion économique.

Enfin, les récents mécanismes de contagion financière marquent une sensibilité accrue des économies émergentes à des chocs associés aux mouvements

\footnotetext{
${ }^{5}$ II s'agit de l'indice composite S\&P/IFCG (IFGDCM\$(PI)) issu de DATASTREAM.

${ }^{6}$ Notons que le manque de données contraint à limiter l'échantillon des modèles faisant intervenir cette variable externe à la période allant de janvier 1995 à mars 2004. Toutefois cette restriction n'est pas préjudiciable à l'analyse étant donné que les principales crises dans les pays émergents ont débuté à la fin de l'année 1994, au Mexique. Toutes les variations de cet indicateur retracent donc l'effet des crises de la fin de la décennie 90.

${ }^{7}$ L'annexe 2 présente les résultats d'un modèle VAR Structurel sur-identifié établi en suivant la méthode proposée par Sims et Zha (1999) permettant de déterminer l'impact d'un choc de taux d'intérêt aux Etats-Unis sur le taux d'intérêt (R), la masse monétaire $(M)$, la production $(Y)$ et le niveau des prix du pays $(P)$ sur une période de 15 mois. Les restrictions contemporaines imposées sont identiques à celles proposées par les auteurs. Les bandes de probabilité sont estimées à $68 \%$.
} 
internationaux de capitaux, particulièrement en période de crise lorsque la structure de leur système financier et bancaire est fragilisée (Corsetti, Pesenti, Roubini,1999) et que le risque d'illiquidité est élevé (Chang et Velasco, 2000). En effet, la perte de confiance des prêteurs internationaux, à la suite d'une crise dans un pays, peut engendrer une réallocation de portefeuille de ces agents en vue de limiter leur exposition au risque dans la région, ayant pour conséquence une sortie massive de capitaux du pays. Cette situation entraîne une baisse de fonds prêtables et de réserves internationales venant déstabiliser le taux de change réel, d'où une augmentation du taux d'intérêt domestique notamment dans les pays ayant adopté un régime de change fixe. Le manque de capitaux étrangers auquel s'ajoute la croissance du taux d'intérêt peut alors créer une baisse importante de la production.

Toutes les variables ont été transformées en logarithme, sauf le taux d'intérêt des USA et des pays du Mercosur. En ce qui concerne les variables domestiques, notons que l'indisponibilité des données portant sur l'indice de production du Paraguay et de l'Uruguay a nécessité une interpolation préalable en vue de passer d'une base trimestrielle à une périodicité mensuelle. Toutes les variables retenues sont désaisonnalisées. Les variables du modèle sont stationnaires, certaines en niveau, d'autres en différence première (annexe 3, tableaux A.3.1 et A.3.2). Cette stationnarité est indispensable pour poser des restrictions de long terme. Enfin, les variables ne sont pas cointégrées.

\section{Les restrictions contemporaines et de long terme}

Notre objectif est d'identifier les $n^{2}$ éléments de la matrice $P$. Statistiquement nous pouvons poser 21 contraintes identifiantes. En effet, comme la matrice $\Omega$ est symétrique, $n(n+1) / 2$ contraintes d'orthogonalisation sont déjà admises. En vue de déterminer les $n(n-1) / 2$ contraintes restantes, 15 dans notre modèle, nous avons utilisé la littérature économique.

Parmi les restrictions les plus souvent imposées dans les modèles VAR Structurels, on retrouve l'hypothèse faisant référence au modèle IS-LM de Gali (1992) et Clarida et Gali (1994) selon laquelle les chocs monétaires sont supposés n'avoir aucun effet de long terme sur le secteur réel. En référence à ces travaux, nous supposons dans notre analyse que les deux chocs monétaires et le choc financier domestiques n'ont pas d'effet sur la production et que les deux chocs monétaires domestiques n'ont pas d'impact sur le taux de change réel dans le long terme.

Par ailleurs, les variables externes sont considérées comme exogènes (Cushman et Zha, 1997). Cette condition concerne en particulier les économies émergentes qui dépendent de manière importante de la situation des marchés internationaux, sans influencer significativement leurs fluctuations (Mackowiack, 2006).

Enfin, de nombreux auteurs (Sims et Zha, 1999; Kim et Roubini, 2000) ont défini leur fonction d'offre de monnaie qui correspond à la fonction de réaction des autorités monétaires c'est à dire au taux d'intérêt sans prendre en compte l'influence des prix et de la production dans le court terme. Cette démarche repose sur l'hypothèse selon laquelle il est indispensable de prendre en compte un délai de réaction de la politique monétaire lié à un manque d'informations. Dans le cadre de notre analyse, la variable prix n'intervient pas. Donc, une seule restriction de court terme est posée concernant la réaction du taux d'intérêt à un choc de production. De la même manière que Kim et Roubini (2000), nous travaillons sur une périodicité 
mensuelle. Nous supposons donc que la réponse de la politique monétaire est différée d'un mois, et non d'un trimestre à la manière de Sims et Zha (1999) ce qui nous paraît plus précis.

La méthodologie de Lack et Lenz (2000) à partir du logiciel RATS a été retenue car elle permet de poser à la fois des restrictions de court et de long terme ${ }^{8}$.

En ce qui concerne les contraintes de court terme, le modèle faisant intervenir tour à tour une variable externe, il s'agit d'une part de poser 4 contraintes visant à souligner l'exogénéité de la variable. En outre, on suppose qu'un choc d'output n'a pas d'impact immédiat sur l'offre de monnaie dans chaque économie. De ce fait: $P_{13}=P_{14}=P_{15}=P_{16}=P_{62}=0$

Au niveau des restrictions de long terme, l'hypothèse d'exogénéité permet d'imposer 5 contraintes supplémentaires. De plus, nous posons l'hypothèse d'un effet nul sur la production des deux chocs monétaires du choc financier ainsi que des deux chocs monétaires sur le taux de change réel, dans le long terme, qui correspond à 5 restrictions supplémentaires.

L'évaluation des multiplicateurs dynamiques issus de la représentation moyenne mobile structurelle du VAR permet d'identifier les réponses structurelles $\varepsilon_{t}$.

Sur la période $s \leq t$, on a $\Theta_{u v}(1)=\lim _{t \rightarrow+\infty} \sum_{j=0}^{t-s} \Theta_{u v, j}$ avec $\Theta_{u v}^{(s)}=\frac{\partial \Delta y_{u t-j}}{\partial \varepsilon_{v s}}$ caractérisant la réponse de long terme d'un choc $\varepsilon_{\mathrm{v}}$ sur une variables $\Delta y_{u}{ }^{9}$, ce qui nous permet de connaître, pour les différentes valeurs de $s$, quel est l'impact d'un choc en $t$ - $s$ sur une des variables en $t$. On pose donc $\Theta_{u v}(1)=(C(1) P)_{u v}=0$ pour $(u, v)=(1,2),(1,3),(1$, $4),(1,5),(1,6),(2,4),(2,5),(2,6),(3,5),(3,6)$.

En suivant les critères d'information de Schwartz, Akaike et Hannan-Quinn nous avons retenu deux retards pour tous les modèles mis à part ceux faisant intervenir la variable EMEl pour lesquels un seul retard suffit. En outre, des tests complémentaires permettent de juger de l'absence d'auto-corrélation des résidus ${ }^{10}$.

La totalité des restrictions requises étant imposée, il est maintenant possible d'identifier les innovations structurelles.

\section{LES EFFETS DES CHOCS EXTERNES SUR CHAQUE PAYS DU MERCOSUR}

Le régime de change en place est un paramètre indispensable à prendre en compte lors de l'analyse de la réponse des pays à des chocs externes, car il représente l'un des principaux déterminants de l'orientation des politiques économiques. Sur la période retenue, l'Argentine qui avait opté pour un currency board, en place jusqu'à la fin de l'année 2001, est le seul pays dont le taux de change a connu une fixité parfaite. On peut s'attendre à ce que le Chili ayant choisi un crawling peg avec une parité fixe vis-à-vis du dollar US ou d'un panier de monnaies en place jusqu'au début

\footnotetext{
${ }^{8}$ Notons que la seule contrainte est que la matrice des restrictions ait une forme triangulaire supérieure. C'est pourquoi, une restriction de court terme concernant l'exogénéité de la variable externe a été supprimée. Cette action n'a cependant pas d'effets quant à la pertinence de l'analyse, dans la mesure où celle-ci n'a pas vocation à étudier les réactions de cette variable à d'éventuels chocs domestiques.

${ }^{9} u$ et $v$ représentent respectivement le nombre de variables et le nombre de chocs.

${ }^{10}$ Les informations concernant le détail des calculs sont disponibles auprès de l'auteur, sur simple demande.
} 
de 1999, et l'Uruguay ayant préféré un système de bandes fixes de 1994 à 1996, puis de crawling band partiellement dollarisé, répondent de manière proche de l'Argentine à des chocs externes communs. A l'inverse, il est difficile d'anticiper les réactions des économies de la Bolivie, du Brésil et du Paraguay du fait de leur préférence pour des régimes flexibles hybrides (de flottement impur ou encadrés par de larges bandes). En effet, dans le cadre de ces régimes de change intermédiaires, les objectifs poursuivis ne sont pas clairement définis, les pays pouvant choisir en cas de choc venant déstabiliser leur économie, entre l'intervention sur le marché des changes dans le but de défendre la stabilité de leur monnaie, et la dévaluation. Par ailleurs, on note que le Brésil a augmenté son taux d'intérêt de manière importante, au début de la décennie quatre-vingt-dix en vue de juguler l'inflation très élevée dans le pays. De ce fait, la réaction du taux d'intérêt du pays est particulièrement forte à la suite de perturbations externes.

Nous pouvons juger de la significativité de nos résultats à partir des graphiques des réponses des pays à chaque choc externe ${ }^{11}$ (annexe 4 , graphiques A.4.1 à A.4.3). Ils reflètent la réaction des variables domestiques qui succède à une variation d'un écart type de la variable externe. L'intervalle de confiance calculé à partir de la procédure boostrapping est de $90 \%$, (Runkle, 1987) $)^{12}$.

Nous allons envisager en premier lieu, un choc relatif au commerce international, à l'origine de bouleversements dans la production et l'exportation des pays d'Amérique Latine. En effet, une variation du prix des produits agricoles (wprices) explique prés de $20 \%$ des fluctuations de l'output $(y)$ dans les pays les plus sensibles, sur une période de deux ans (annexe 4, tableau A.4.1). Cette perturbation correspond à un choc de demande réelle de produits agricoles en provenance de la zone Mercosur. Une hausse de ce prix a pour conséquence une augmentation significative de la production de chacun des pays de la zone qui bénéficient d'avantages comparatifs dans le secteur agricole, en particulier en Argentine, au Brésil et au Paraguay où les effets bénéfiques sur la production sont comparables. Les résultats sont nettement moins significatifs au Chili où la production et l'exportation de biens agricoles a une importance décroissante ces dernières années par rapport au secteur secondaire et tertiaire. De même, en Uruguay, la hausse du prix des produits agricoles n'a pas eu l'effet bénéfique attendu sur la production. Les conditions climatiques défavorables qu'a connues le pays ainsi que son manque de compétitivité par rapport à ses voisins (suite à la dévaluation du real brésilien par exemple) peuvent expliquer cette situation. Cependant, la réaction du taux de change réel (rerte) des pays est faible car la plupart des exportations sont libellées en dollars US. Par ailleurs, au Brésil, la demande de monnaie $(m)$ étant particulièrement sensible aux variations de l'output (l'élasticité de la demande de monnaie par rapport à l'output est de 2,1), une croissance de la production provoque une augmentation de la masse monétaire les six premiers mois, limitée ensuite par la hausse du taux d'intérêt.

\footnotetext{
${ }^{11}$ Notons que la réaction d'une variable à un choc est significative dès lors que l'axe des abscisses n'est pas inclus dans l'intervalle de confiance qui encadre la réaction de cette variable.

${ }^{12}$ Dans le cadre de l'étude de l'impact des chocs externes sur les économies du Mercosur, nous sommes en présence d'un modèle juste identifié dans lequel apparaissent des contraintes de court et long terme. Dans ce cas, la procédure boostrapping et l'approche bayésienne de Sims et Zha (1999) pour calculer les bandes d'erreur sont équivalentes. C'est pourquoi la méthode proposée par Lack et Lenz (2000) a été privilégiée. Le degré de précision de nos résultats est garanti par le choix d'un intervalle de confiance à $90 \%$, rarement retenu dans les études fondées sur un VAR structurel qui retiennent généralement un écart-type autour de la moyenne.
} 
Le choc positif de taux d'intérêt aux Etats-Unis (usintrte) a un impact immédiat sur le taux d'intérêt des pays considérés (ninrte). II est à l'origine d'une fluctuation de ces taux proche de $30 \%$ dès le début de la période pour la majorité des pays (annexe 4 , tableau A.4.2). Plusieurs facteurs sont à l'origine de ce phénomène. Tout d'abord, la plupart des pays du Mercosur, durant la plus grande partie de la période considérée avaient opté pour un régime de change plutôt fixe par rapport au dollar US. En outre, la petite taille du pays est en partie responsable de sa sensibilité vis-à-vis des chocs externes. Par ailleurs, limiter l'écart des taux d'intérêt par rapport aux Etats-Unis permet aux pays de conserver la confiance des marchés financiers et ainsi de préserver la stabilité du régime. Cette augmentation est significative dans la majorité des pays sur toute la période considérée en Bolivie et dans le plus court terme en Argentine, au Brésil et au Paraguay. Au Brésil le taux d'intérêt réagit très fortement pour lutter contre l'inflation. En revanche, la variation du taux d'intérêt domestique est limitée en Argentine à celle du taux d'intérêt américain du fait de la dépendance de la politique monétaire domestique vis-à-vis de la politique monétaire américaine pendant la période du currency board. Les réactions de la politique monétaire de I'Uruguay et du Chili sont peu significatives. En ce qui concerne le Chili, nos résultats convergent avec ceux de Mackowiak (2006), qui justifie le fait que l'économie du Chili réagit très peu à un choc de taux d'intérêt américain grâce à une politique de change bien encadrée. En Uruguay, la faible montée des taux d'intérêt ne permet pas de limiter la sortie des capitaux du pays (asset). L'Uruguay est donc très fragilisé à la suite d'un choc de politique monétaire américaine restrictive.

Cette augmentation du taux d'intérêt ne se répercute que faiblement sur la masse monétaire $(m)$, hormis en Uruguay où elle augmente du fait d'une réaction du taux d'intérêt domestique insuffisante. En ce sens, nos conclusions rejoignent celles de Mackowiak (2006). En outre, un taux d'intérêt domestique élevé limite la sortie de capitaux dans la plupart des pays à l'exception de l'Uruguay. Par conséquent, les effets sur le taux de change réel (rerte) sont faibles, de même que ceux sur la production $(y)$. Effectivement, d'une part la transmission des chocs par le canal commercial au bénéfice du pays ne fonctionne pas, du fait de la dépréciation insuffisante du taux de change. D'autre part, l'augmentation des taux d'intérêt domestiques ne semble pas être un frein particulier à la croissance car elle permet de conserver un certain montant d'actifs dans l'économie. Sur ce point, nos résultats sont proches de ceux de Canova (2003).

Face à une volatilité accrue de l'indice boursier composite des pays émergents (emei), les pays du Mercosur subissent la perte de confiance des investisseurs internationaux, particulièrement significative au Paraguay qui connaît une importante sortie de capitaux étrangers (asset). Ce phénomène se traduit par une dépréciation de la monnaie (rerte) sur toute la période considérée en Bolivie et sur le plus court terme dans les autres pays, sauf en Argentine où la réaction du taux de change est non significative. Pour limiter les effets néfastes de ce choc, les banques centrales du Chili, du Paraguay et de l'Uruguay interviennent sur le taux d'intérêt domestique (ninrte) durant les premiers mois qui suivent cette perturbation. Par ailleurs elles peuvent, à l'aide d'une politique discrétionnaire, échanger des devises contre de la monnaie nationale en vue de limiter la dévaluation du taux de change nominal, préjudiciable pour la crédibilité du pays sur les marchés financiers. Ainsi, ces facteurs peuvent expliquer l'importante contraction de la masse monétaire $(m)$ de la plupart des pays de la zone, l'Argentine mise à part. Cependant, bien que la dépréciation du taux de change réel puisse rendre les pays plus compétitifs en ce qui 
concerne les échanges internationaux, celle-ci ne permet pas de compenser l'effet de la montée des taux d'intérêt et de la diminution de la masse monétaire ayant pour conséquence une large baisse de la production $(y)$ les mois suivants. Ainsi, un choc de volatilité de l'indice boursier composite explique entre $11 \%$ et $27 \%$ de la fluctuation du taux d'intérêt respectivement dans les premiers mois qui suivent le choc, prés de $30 \%$ de la variation du taux de change réel pour certains pays, et une large part de la chute de la production (plus de $30 \%$ pour la majorité des pays l'année suivant le choc), (annexe 4, tableau A.4.3). Toutefois, les variations macroéconomiques sont réduites en Argentine du fait de la rigueur due à la dépendance de la politique monétaire et de change du pays et des Etats-Unis dans le cadre du currency board. En revanche, l'Argentine, comme les autres pays, voit sa croissance économique affectée. En ce sens, on en déduit que les effets néfastes sur la production sont équivalents quel que soit le régime de change en place. Seule la politique de change très encadrée du Chili permet de limiter ces effets. L'analyse de l'impact d'un choc de volatilité de l'indice boursier composite émergent dans les pays du Mercosur, permet donc de souligner la sensibilité de ces pays face aux variations financières internationales qui se propagent de la sphère financière à la sphère réelle de l'économie.

\section{ANALYSE DE LA CORRELATION DES REPONSES AUX CHOCS}

En vue de comparer les réactions des pays de la zone face à des chocs externes, nous avons procédé à une analyse des co-mouvements des réponses significatives des pays (annexe 5, tableaux A.5.1 à A.5.5).

Les réponses de l'Argentine, du Brésil et du Paraguay dont la production est majoritairement agricole et axée sur l'exportation sont largement corrélées à la suite d'un choc de prix des produits agricoles (wprices). Par ailleurs, il existe de nombreuses similitudes entre les pays qui ont répondu par une hausse de leur taux d'intérêt à un choc de taux d'intérêt des USA (usintrte), le Chili et l'Uruguay mis à part. En ce qui concerne un choc de l'indice boursier des pays émergents (emei), tous les pays, excepté le Chili, subissent la perte de confiance des marchés qui se traduit par une baisse des actifs du pays à l'origine d'une large dépréciation taux de change réel (rerte). En ce sens, la réaction de tous les pays est largement corrélée. II s'en suit une augmentation significative des taux d'intérêt (ninrte) durant les six premiers mois qui suivent le choc, sauf en Bolivie et en Argentine où cette augmentation est modérée en raison de la forte dépendance du pays vis-à-vis de la politique monétaire américaine et au Brésil ou la variable répond de manière moins marquée. Cette réaction des taux d'intérêt engendre une diminution importante de la masse monétaire $(m)$ largement corrélée entre tous les pays. Enfin, la baisse de la production $(y)$ qui en découle est fortement corrélée au sein de la zone. Ces résultats montrent que les économies souffrent d'un défaut de soutenabilité de leur régime de change durant la période retenue. Ainsi, aucune solution de change ne semble avoir été plus efficace pour limiter les effets négatifs d'un tel choc.

Toutefois, même s'il existe des similitudes dans la réaction des pays, notamment à la suite d'un choc de confiance des marchés, le plus souvent leurs réponses divergent. Seuls le Paraguay et le Brésil ainsi que de la Bolivie et l'Uruguay réagissent de manière proche à chaque type de choc. Par ailleurs, les fluctuations économiques de 
la Bolivie et de Brésil sont voisines en cas de choc de prix des produits agricoles et de taux d'intérêt américain. On en déduit donc que la taille des économies n'influence pas leur réaction à des chocs communs.

\section{CONCLUSION}

A partir d'un modèle VAR Structurel, qui nous a permis d'imposer des restrictions contemporaines et de long terme sur une base théorique, nous avons étudié les réactions des pays du Mercosur à des chocs externes, en vue de voir dans quelles mesures ces perturbations ont été à l'origine de la déstabilisation des économies durant la dernière décennie. II ressort de notre analyse, une importante vulnérabilité des pays à des chocs internationaux de prix des produits agricoles, du taux d'intérêt US et de la volatilité des indices boursiers des pays émergents. Ce phénomène met en avant le manque de soutenabilité des régimes de change en place durant cette période du fait notamment de la faible influence des pays sur la scène internationale et de leur manque de crédibilité sur les marchés financiers. Ainsi, mis à part au Chili, aucune solution de change plus ou moins fixe dans cette région ne pouvait être envisagée par les pays. Par ailleurs, il n'existe pas un noyau de pays dont les réactions à chaque type de choc soient identiques. Cette situation ne semble dépendre ni de la taille des économies, ni du régime de change en place. Par conséquent, il est clair que les causes de l'asymétrie des réponses des pays du Mercosur à un choc commun proviennent d'un manque de convergence des structures macroéconomiques et financières entre les pays.

\section{Annexe 1.}

Soit la représentation vectorielle autorégressive $\operatorname{VAR}(q)$ du modèle sous forme réduite :

$$
\Delta Y_{t}=\sum_{i=1}^{q} A_{i} \Delta Y_{t-i}+e_{t}
$$

où $q$ est le nombre de retards, $e_{t}$ est un bruit blanc.

En vue de simplifier notre représentation, les variables sont réparties en deux blocs : $\Delta y_{1 t}$ représente les variables domestiques et $\Delta y_{2 t}$ les variables internationales, où $\Delta y_{1 t}$ et $\Delta y_{2 t}$ sont les différences premières entre $t-1$ et $t$ de $y_{1 t}$ et $y_{2 t}$. II en est de même pour le terme d'erreur $e_{t}$.

On a donc : 
$\Delta Y_{t}=\left(\begin{array}{l}\Delta y_{1 t} \\ \Delta y_{2 t}\end{array}\right) \quad$ avec $\Delta Y_{t-1}$ le vecteur de ces variables retardées, $A_{i}=\left(\begin{array}{ll}a_{11}^{(i)} & a_{12}^{(i)} \\ a_{21}^{(i)} & a_{22}^{(i)}\end{array}\right)$ la matrice $n \times n$ des paramètres du modèle, $e_{t}=\left(\begin{array}{l}e_{1 t} \\ e_{2 t}\end{array}\right) \quad \begin{aligned} & \text { le vecteur d'erreur dont la matrice des variances covariances ne fait } \\ & \text { l'objet d'aucune restriction, c'est-à-dire } E\left(e_{t}, e_{t}^{T}\right)=\Omega \text { et } E\left(e_{t}\right)=0 .\end{aligned}$

Le modèle $\operatorname{VAR}(q)$ peut s'écrire alors sous la forme :

$$
\begin{aligned}
& A(L) \Delta Y_{t}=e_{t} \quad \text { où } \\
& A(L)=I_{n}-A_{1} L-\ldots-A_{q} L^{q}
\end{aligned}
$$

On suppose que $\Delta Y_{t}$ est stationnaire, $A(L)$ est donc inversible ${ }^{13}$, c'est à dire qu'il existe $n \times n$ paramètres de la matrice $C_{j}$ avec $C_{0}=I_{n}$ et $\sum_{j=0}^{\infty} C_{j} C_{j}{ }^{T}$ est une matrice finie de telle sorte qu'on ait : $A(L)^{-1}=C(L)$

avec

$$
C(L)=I_{n}+C_{1} L+C_{2} L^{2}+\ldots=\sum_{j=0}^{\infty} C_{j} L^{j}
$$

Le VAR canonique sous forme moyenne mobile se présente donc sous la forme :

$$
\Delta Y_{t}=\sum_{j=0}^{\infty} C_{j} e_{t-j}=C(L) e_{t}
$$

où $e_{t}$ représente le vecteur des innovations canoniques.

On pose la relation selon laquelle le vecteur des innovations canoniques $e_{t}$ est une combinaison linéaire des impulsions structurelles $\varepsilon_{t}$ à la même date. Cela signifie que : $e_{t}=P \varepsilon_{t}$

Pour poser des restrictions de long terme, on passe par l'écriture de la moyenne mobile structurelle du VAR :

$$
P^{-1} \Delta Y_{t}=P^{-1} \sum_{j=0}^{\infty} C_{j} e_{t-j} \Leftrightarrow \Delta Y_{t}=\sum_{j=0}^{\infty} C_{j} P P^{-1} e_{t-j} \Leftrightarrow \Delta Y_{t}=\sum_{j=0}^{\infty} \Theta_{j} \varepsilon_{t-j}=\Theta(L) \varepsilon_{t}
$$

avec $\Theta(L)=P C(L)=P+P C_{1} L+\ldots \quad$ et $\quad \Theta(L)=\sum_{j=0}^{\infty} \Theta_{j} L^{j}$

\footnotetext{
${ }^{13}$ L'exposant $T$ désigne la transposée de la variable concernée.
} 
Ou, suivant une représentation plus détaillée :

$$
\left(\begin{array}{c}
\Delta y_{1} \\
\Delta y_{2}
\end{array}\right)=\sum_{j=0}^{\infty} L^{j}\left(\begin{array}{ll}
\theta_{11}^{(j)} & \theta_{12}^{(j)} \\
\theta_{21}^{(j)} & \theta_{22}^{(j)}
\end{array}\right)
$$

\section{Annexe 2.}

Graphique A.2.1. Réponses des Etats-Unis à un choc de taux d'intérêt US (usintrte)
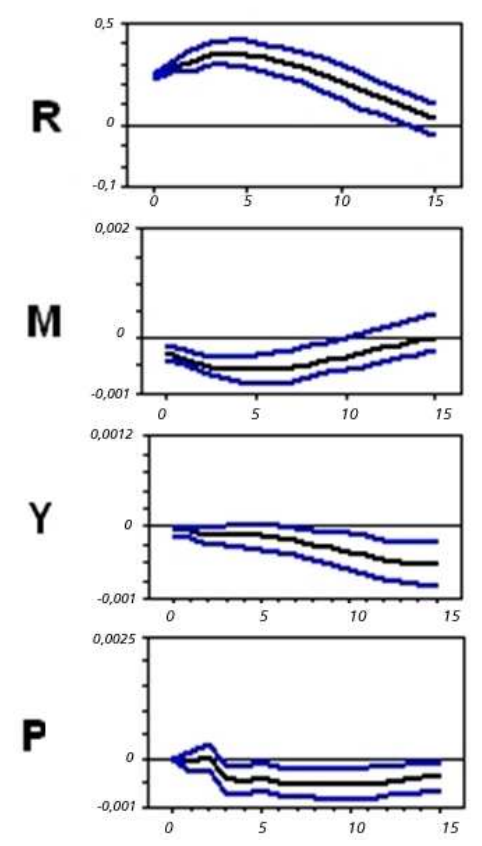

avec $\mathrm{R}$ le taux d'intérêt, $\mathrm{M}$, la masse monétaire, $\mathrm{Y}$ la production et $\mathrm{P}$ le niveau des prix (Sims et Zha,1999)

\section{Annexe 3.}

Tableau A.3.1. Test de racine unitaire Dickey-Fuller des variables externes

\begin{tabular}{|c|c|c|c|c|c|}
\hline wprices & $\Delta$ wprices & usintrte & Ausintrte & emei & $\Delta e m e i$ \\
\hline-2.066 & $-7.130^{\star *}$ & -1.086 & $-5.902^{\star *}$ & -1.891 & $-5.946^{\star *}$ \\
\hline
\end{tabular}

Tableau A.3.2. Test de racine unitaire Dickey-Fuller des variables internes

\begin{tabular}{|c|c|c|c|c|c|c|c|c|c|c|}
\hline & y & $\Delta y$ & rerte & $\Delta$ rerte & asset & $\Delta$ asset & $m$ & $\Delta m$ & ninrte & $\Delta$ ninrte \\
\hline Argentine & -2.044 & $-7.429^{\star \star}$ & -2.233 & $-6.698^{* *}$ & $-3.951^{*}$ & $-10.62^{* *}$ & $-3.616^{*}$ & $-7.919^{\star \star}$ & $-11.30^{\star *}$ & $-13.51^{\star *}$ \\
\hline Bolivie & -1.774 & $-9.621^{\star *}$ & -0.384 & $-7.935^{\star *}$ & -2.532 & $-8.896^{\star \star}$ & -1.918 & $-10.29^{\star *}$ & -2.858 & $-11.35^{\star \star}$ \\
\hline Brésil & -1.315 & $-9.002^{* *}$ & -1.847 & $-9.372^{* *}$ & $-5.589^{* *}$ & $-10.06^{* *}$ & -1.905 & $-7.523^{\star \star}$ & $-3.626^{*}$ & $-9.942^{* *}$ \\
\hline Chili & -2.191 & $-7.136^{\star *}$ & -1.969 & $-8.910^{\star *}$ & $-4.228^{\star *}$ & $-9.223^{\star *}$ & -2.304 & $-12.17^{\star \star}$ & $-6.926^{\star \star}$ & $-10.72^{\star \star}$ \\
\hline Paraguay & -0.994 & $-6.408^{* *}$ & -1.642 & $-8.857^{* *}$ & -2.357 & $-8.022^{* *}$ & -1.853 & $-6.449^{* *}$ & $-4.972^{* *}$ & $-14.11^{* *}$ \\
\hline Uruguay & -0.641 & $-7.640^{\star *}$ & -1.261 & $-9.417^{\star \star}$ & $-3.542^{*}$ & $-13.77^{\star \star}$ & -1.531 & $-8.286^{\star \star}$ & -3.122 & $-11.74^{\star \star}$ \\
\hline
\end{tabular}

Nous avons procédé à 1 retard pour la totalité des tests, nous avons pris en compte la constante et le trend. 


\section{Annexe 4}

Graphique A.4.1. Réponses des pays à un choc de prix des matières premières (wprices)
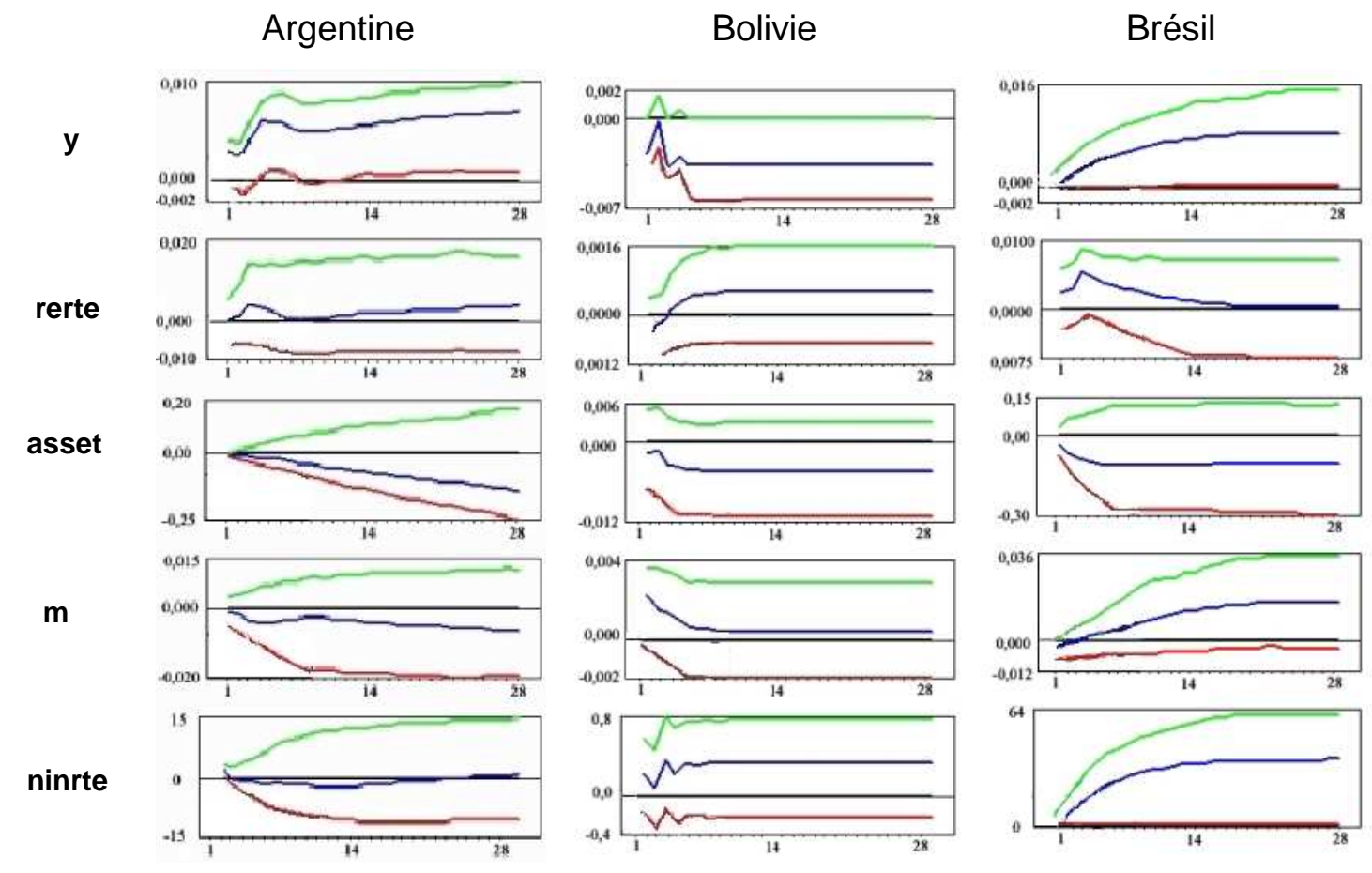

Chili

Paraguay

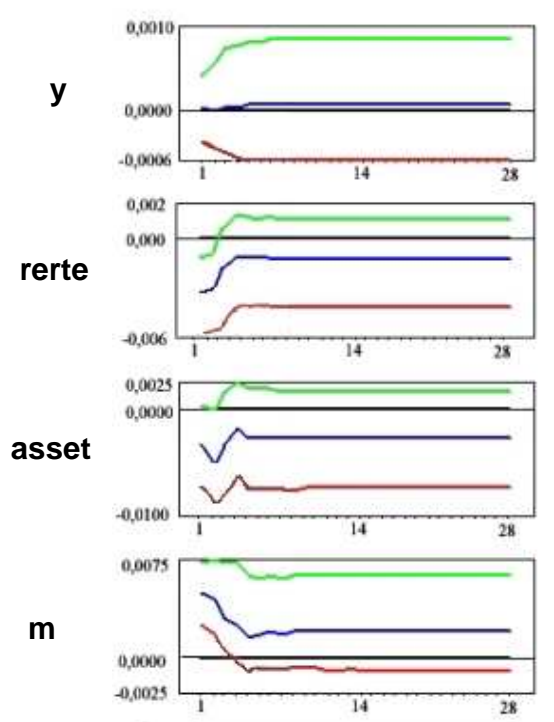

ninrte

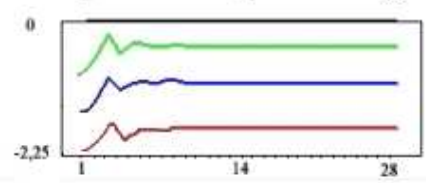

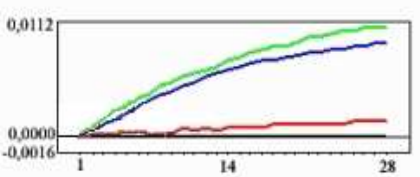
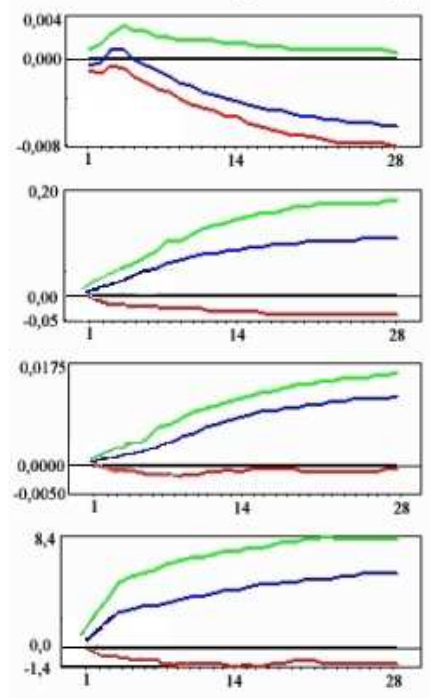

Uruguay
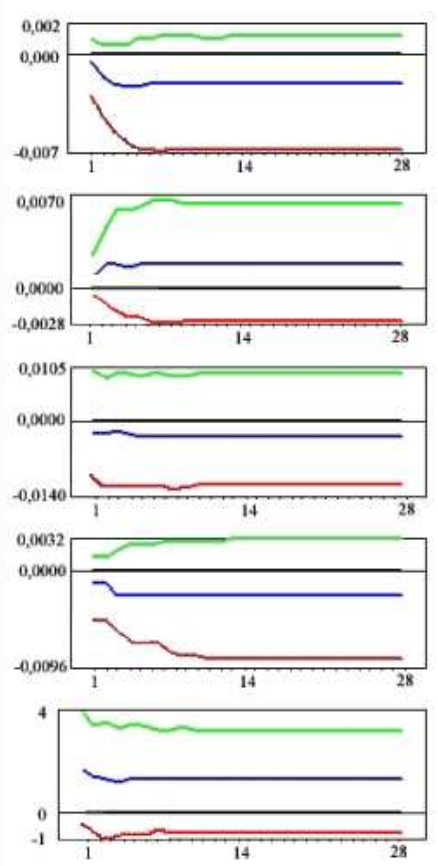
Tableau A.4.1. Décomposition de la variance à la suite d'un choc de prix des matières premières (wprices)

\begin{tabular}{|c|c|c|c|c|c|c|}
\hline Choc & Argentine & Bolivie & Brésil & Chili & Paraguay & Uruguay \\
\hline \multicolumn{7}{|l|}{ y } \\
\hline 1 & 15,18 & 3,93 & 0,62 & 0,04 & 0,01 & 0,53 \\
\hline 2 & 11,37 & 2,56 & 1,01 & 0,01 & 1,81 & 1,54 \\
\hline 4 & 20,14 & 5,01 & 2,76 & 0,02 & 3,63 & 2,17 \\
\hline 8 & 20,51 & 6,85 & 5,79 & 0,04 & 9,56 & 2,39 \\
\hline 14 & 21,13 & 7,72 & 9,31 & 0,04 & 15,26 & 2,48 \\
\hline 28 & 25,91 & 8,30 & 13,16 & 0,04 & 20,90 & 2,54 \\
\hline \multicolumn{7}{|l|}{ rerte } \\
\hline 1 & 0,02 & 0,70 & 0,74 & 10,76 & 0,14 & 0,69 \\
\hline 2 & 0,24 & 0,69 & 1,17 & 9,70 & 0,07 & 1,40 \\
\hline 4 & 0,96 & 0,45 & 2,72 & 5,80 & 0,69 & 1,32 \\
\hline 8 & 0,46 & 0,92 & 2,34 & 3,66 & 0,82 & 1,23 \\
\hline 14 & 0,29 & 1,23 & 1,51 & 2,81 & 3,70 & 1,21 \\
\hline 28 & 0,46 & 1,43 & 0,76 & 2,23 & 8,77 & 1,20 \\
\hline \multicolumn{7}{|l|}{ asset } \\
\hline 1 & 3,81 & 0,36 & 3,98 & 3,39 & 3,31 & 0,13 \\
\hline 2 & 2,14 & 0,27 & 4,38 & 5,01 & 2,71 & 0,17 \\
\hline 4 & 1,57 & 1,17 & 4,79 & 3,53 & 2,95 & 0,21 \\
\hline 8 & 2,29 & 2,11 & 4,61 & 2,74 & 4,73 & 0,34 \\
\hline 14 & 3,55 & 2,60 & 4,20 & 2,42 & 5,75 & 0,40 \\
\hline 28 & 4,60 & 2,93 & 3,73 & 2,21 & 6,08 & 0,44 \\
\hline \multicolumn{7}{|l|}{$m$} \\
\hline 1 & 0,09 & 3,14 & 3,24 & 7,57 & 0,23 & 0,71 \\
\hline 2 & 0,29 & 2,80 & 2,67 & 8,79 & 0,14 & 0,73 \\
\hline 4 & 0,98 & 2,08 & 1,54 & 6,53 & 0,11 & 1,27 \\
\hline 8 & 0,71 & 1,19 & 1,31 & 4,55 & 1,25 & 1,34 \\
\hline 14 & 0,52 & 0,75 & 3,95 & 3,69 & 4,47 & 1,37 \\
\hline 28 & 0,84 & 0,45 & 8,79 & 3,06 & 8,58 & 1,40 \\
\hline \multicolumn{7}{|l|}{ ninrte } \\
\hline 1 & 4,94 & 0,42 & 29,33 & 12,84 & 7,43 & 1,45 \\
\hline 2 & 1,93 & 0,33 & 30,38 & 12,46 & 5,09 & 1,93 \\
\hline 4 & 1,05 & 0,89 & 29,67 & 10,84 & 4,54 & 1,99 \\
\hline 8 & 0,49 & 1,37 & 29,08 & 9,70 & 4,63 & 2,19 \\
\hline 14 & 0,44 & 1,64 & 28,59 & 9,14 & 4,89 & 2,32 \\
\hline 28 & 0,18 & 1,84 & 28,03 & 8,75 & 5,89 & 2,42 \\
\hline
\end{tabular}


Graphique A.4.2. Réponses des pays à un choc de taux d'intérêt US (usintrte)
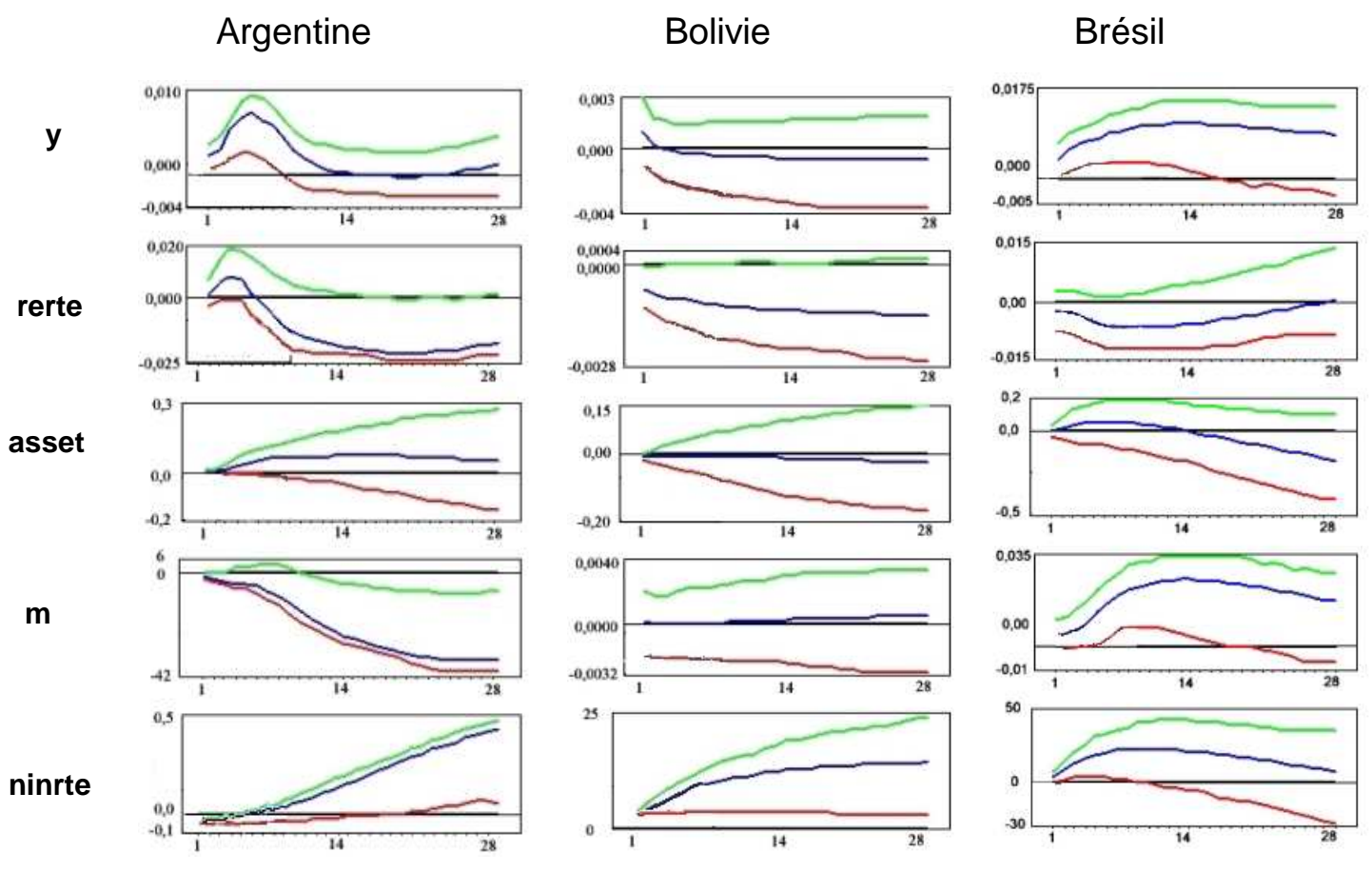

\section{Chili}
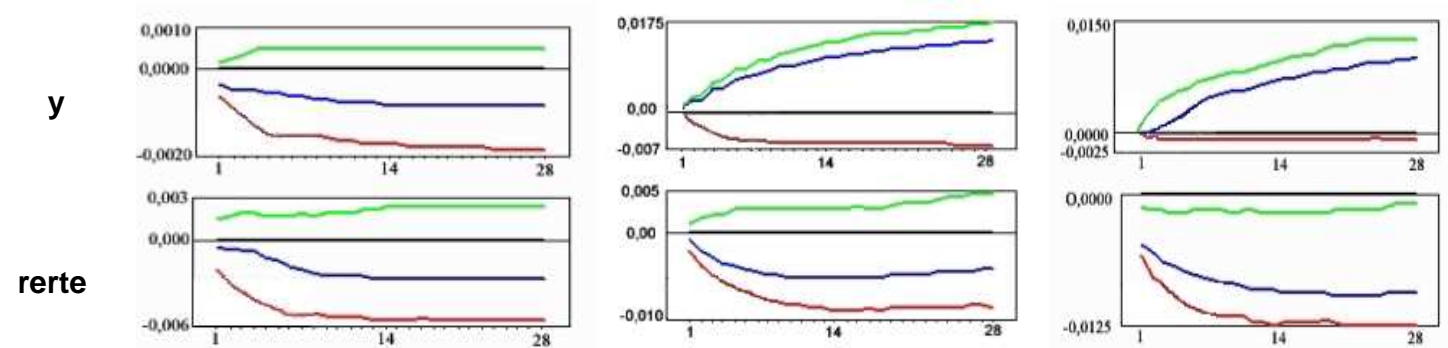

asset
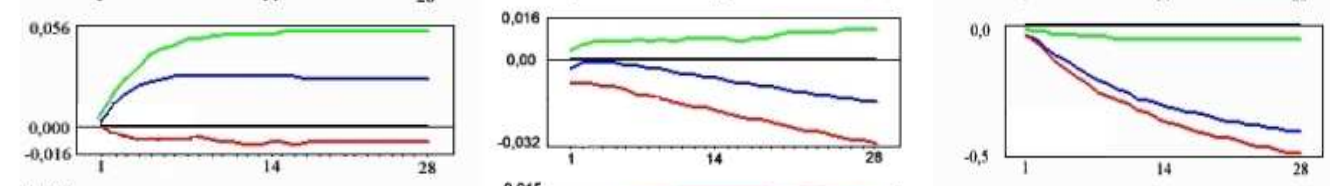

m
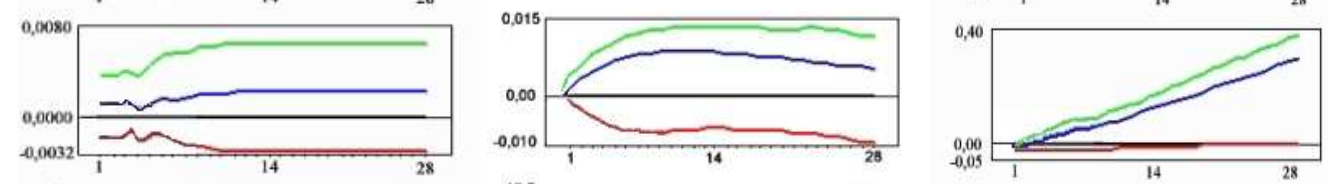

ninrte
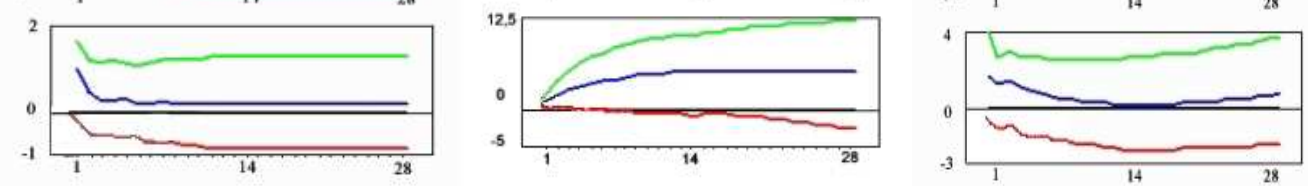
Tableau A.4.2. Décomposition de la variance à la suite d'un choc de taux d'intérêt US (usintrte)

\begin{tabular}{|c|c|c|c|c|c|c|}
\hline Choc & Argentine & Bolivie & Brésil & Chili & Paraguay & Uruguay \\
\hline \multicolumn{7}{|l|}{$y$} \\
\hline 1 & 8,54 & 0,33 & 3,12 & 3,03 & 19,8 & 0,29 \\
\hline 2 & 10,67 & 0,23 & 6,70 & 3,57 & 18,05 & 0,33 \\
\hline 4 & 25,79 & 0,14 & 8,70 & 3,10 & 21,16 & 1,56 \\
\hline 8 & 29,63 & 0,15 & 14,7 & 3,50 & 23,08 & 4,52 \\
\hline 14 & 21,07 & 0,22 & 15,6 & 4,57 & 23,94 & 8,69 \\
\hline 28 & 13,95 & 0,32 & 20,9 & 6,00 & 24,75 & 15,72 \\
\hline \multicolumn{7}{|l|}{ rerte } \\
\hline 1 & 1,12 & 4,17 & 0,9 & 0,10 & 0,53 & 16,71 \\
\hline 2 & 6,76 & 4,09 & 1,28 & 0,16 & 1,99 & 14,56 \\
\hline 4 & 8,17 & 4,41 & 2,06 & 0,29 & 2,91 & 16,12 \\
\hline 8 & 6,28 & 5,28 & 4,24 & 1,42 & 3,41 & 19,20 \\
\hline 14 & 21,38 & 6,29 & 5,58 & 3,10 & 3,45 & 22,45 \\
\hline 28 & 33,95 & 7,30 & 3,86 & 4,53 & 3,55 & 25,76 \\
\hline \multicolumn{7}{|l|}{ asset } \\
\hline 1 & 0,03 & 0,22 & 0,24 & 7,87 & 0,83 & 37,75 \\
\hline 2 & 1,53 & 0,08 & 0,74 & 12,67 & 1,11 & 42,00 \\
\hline 4 & 9,21 & 0,02 & 1,35 & 13,49 & 1,19 & 45,18 \\
\hline 8 & 11,83 & 0,01 & 1,36 & 13,03 & 1,29 & 46,26 \\
\hline 14 & 7,41 & 0,04 & 0,79 & 12,08 & 1,50 & 46,40 \\
\hline 28 & 3,41 & 0,17 & 2,36 & 11,19 & 1,90 & 46,46 \\
\hline \multicolumn{7}{|l|}{$m$} \\
\hline 1 & 1,96 & 0,03 & 1,49 & 0,00 & 0,52 & 7,02 \\
\hline 2 & 3,94 & 0,02 & 1,33 & 0,15 & 2,82 & 7,43 \\
\hline 4 & 8,58 & 0,01 & 3,26 & 0,26 & 3,49 & 9,26 \\
\hline 8 & 12,12 & 0,01 & 12,9 & 0,81 & 3,96 & 13,39 \\
\hline 14 & 37,06 & 0,02 & 23,22 & 1,54 & 3,97 & 18,97 \\
\hline 28 & 52,78 & 0,07 & 26,05 & 2,12 & 4,22 & 27,12 \\
\hline \multicolumn{7}{|l|}{ ninrte } \\
\hline 1 & 9,51 & 32,46 & 15,77 & 6,50 & 14,13 & 1,47 \\
\hline 2 & 5,56 & 34,00 & 19,07 & 4,74 & 16,22 & 1,62 \\
\hline 4 & 1,66 & 35,44 & 22,13 & 3,18 & 16,26 & 1,66 \\
\hline 8 & 3,04 & 35,46 & 21,17 & 2,28 & 16,40 & 1,24 \\
\hline 14 & 19,35 & 32,73 & 16,68 & 1,62 & 16,51 & 0,79 \\
\hline 28 & 37,99 & 24,82 & 9,53 & 1,11 & 16,52 & 0,50 \\
\hline
\end{tabular}


Graphique A.4.3. Réponses des pays à un choc de volatilité boursière (emei)
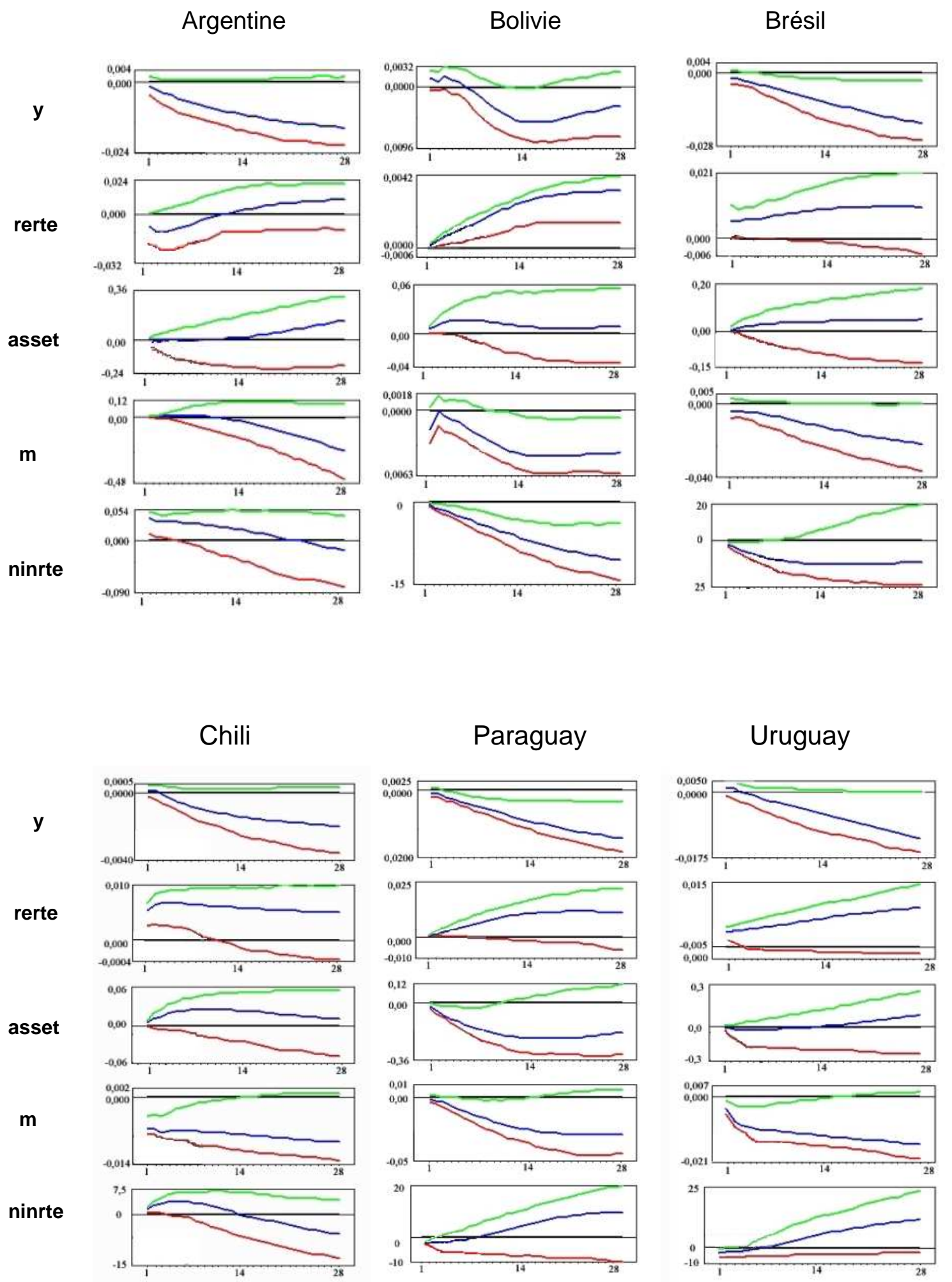
Tableau A.4.3. Décomposition de la variance à la suite d'un choc de volatilité boursière (emei)

\begin{tabular}{|c|c|c|c|c|c|c|}
\hline Choc & Argentine & Bolivie & Brésil & Chili & Paraguay & Uruguay \\
\hline \multicolumn{7}{|l|}{ y } \\
\hline 1 & 1,05 & 1,23 & 0,88 & 0,02 & 4,30 & 0,73 \\
\hline 2 & 2,50 & 1,10 & 1,39 & 0,03 & 5,65 & 0,46 \\
\hline 4 & 6,18 & 1,30 & 2,39 & 0,54 & 13,43 & 0,20 \\
\hline 8 & 13,18 & 0,91 & 5,47 & 4,67 & 31,43 & 0,72 \\
\hline 14 & 23,89 & 4,28 & 12,31 & 11,62 & 52,99 & 2,85 \\
\hline 28 & 33,61 & 5,76 & 31,08 & 22,03 & 76,53 & 10,83 \\
\hline \multicolumn{7}{|l|}{ rerte } \\
\hline 1 & 10,86 & 2,52 & 3,95 & 29,52 & 0,89 & 6,03 \\
\hline 2 & 10,98 & 2,59 & 4,17 & 28,52 & 0,40 & 5,74 \\
\hline 4 & 9,68 & 3,50 & 4,80 & 28,45 & 1,47 & 5,54 \\
\hline 8 & 6,65 & 8,12 & 6,26 & 28,87 & 8,65 & 6,14 \\
\hline 14 & 4,29 & 19,44 & 8,49 & 28,43 & 20,34 & 8,07 \\
\hline 28 & 6,97 & 32,99 & 11,72 & 26,07 & 29,52 & 13,56 \\
\hline \multicolumn{7}{|l|}{ asset } \\
\hline 1 & 0,47 & 7,28 & 3,84 & 6,17 & 54,27 & 2,36 \\
\hline 2 & 0,10 & 7,08 & 3,66 & 8,35 & 54,56 & 2,44 \\
\hline 4 & 0,09 & 6,30 & 3,40 & 10,14 & 54,85 & 1,89 \\
\hline 8 & 0,11 & 3,99 & 3,03 & 10,52 & 53,75 & 0,94 \\
\hline 14 & 0,21 & 1,84 & 2,72 & 9,16 & 49,98 & 0,33 \\
\hline 28 & 3,17 & 0,64 & 2,77 & 5,57 & 38,43 & 0,68 \\
\hline \multicolumn{7}{|l|}{$m$} \\
\hline 1 & 2,09 & 1,91 & 2,22 & 22,92 & 0,44 & 3,93 \\
\hline 2 & 0,45 & 1,25 & 2,34 & 27,54 & 1,59 & 7,92 \\
\hline 4 & 0,69 & 0,99 & 2,89 & 30,00 & 7,46 & 11,13 \\
\hline 8 & 0,42 & 2,27 & 4,70 & 30,62 & 23,72 & 13,77 \\
\hline 14 & 1,64 & 6,92 & 8,92 & 30,69 & 38,56 & 15,76 \\
\hline 28 & 11,51 & 12,17 & 21,71 & 33,28 & 43,73 & 19,32 \\
\hline \multicolumn{7}{|l|}{ ninrte } \\
\hline 1 & 18,61 & 17,39 & 23,38 & 27,99 & 12,10 & 11,58 \\
\hline 2 & 18,55 & 18,32 & 21,55 & 27,71 & 11,24 & 8,40 \\
\hline 4 & 18,23 & 18,92 & 19,75 & 24,49 & 7,52 & 4,37 \\
\hline 8 & 16,47 & 20,17 & 17,44 & 16,19 & 2,89 & 1,32 \\
\hline 14 & 10,71 & 22,56 & 14,62 & 7,72 & 2,67 & 2,30 \\
\hline 28 & 5,20 & 23,77 & 9,99 & 6,93 & 10,60 & 7,26 \\
\hline
\end{tabular}

\section{Annexe 5.}

Tableau A.5.1. Corrélation des réponses de $y$ à différents chocs externes

\begin{tabular}{|c|c|c|c|}
\hline & wprices & usintrte & emei \\
\hline AG-BO & $-0,69$ & 0,48 & 0,82 \\
\hline AG-BR & 0,84 & $-0,47$ & 0,98 \\
\hline AG-CL & 0,84 & 0,78 & 0,99 \\
\hline AG-PY & 0,83 & $-0,73$ & 0,99 \\
\hline AG-UY & $-0,59$ & $-0,72$ & 0,98 \\
\hline BO-BR & $-0,64$ & $-0,86$ & 0,73 \\
\hline BO-CL & $-0,66$ & 0,91 & 0,85 \\
\hline BO-PY & $-0,51$ & $-0,87$ & 0,77 \\
\hline BO-UY & 0,43 & $-0,87$ & 0,72 \\
\hline BR-CL & 0,78 & $-0,76$ & 0,96 \\
\hline BR-PY & 0,97 & 0,67 & 0,99 \\
\hline BR-UY & $-0,57$ & 0,63 & 0,99 \\
\hline CL-PY & 0,67 & $-0,97$ & 0,98 \\
\hline CL-UY & $-0,89$ & $-0,97$ & 0,97 \\
\hline PY-UY & $-0,44$ & 0,99 & 0,99 \\
\hline
\end{tabular}


Tableau A.5.2. Corrélation des réponses de rerte à différents chocs externes

\begin{tabular}{|c|c|c|c|}
\hline & wprices & usintrte & emei \\
\hline AG-BO & 0,20 & 0,91 & 0,99 \\
\hline AG-BR & $-0,24$ & $-0,14$ & 0,99 \\
\hline AG-CL & 0,04 & 0,96 & $-0,90$ \\
\hline AG-PY & $-0,44$ & 0,77 & 0,95 \\
\hline AG-UY & 0,39 & 0,92 & 0,97 \\
\hline BO-BR & $-0,53$ & $-0,31$ & 0,99 \\
\hline BO-CL & $-0,03$ & 0,95 & $-0,84$ \\
\hline BO-PY & $-0,62$ & 0,76 & 0,98 \\
\hline BO-UY & 0,42 & 0,98 & 0,96 \\
\hline BR-CL & 0,74 & $-0,14$ & $-0,84$ \\
\hline BR-PY & 0,96 & 0,37 & 0,98 \\
\hline BR-UY & 0,00 & $-0,24$ & 0,96 \\
\hline CL-PY & 0,62 & 0,88 & $-0,72$ \\
\hline PL-UY & 0,16 & 0,97 & $-0,88$ \\
\hline
\end{tabular}

Tableau A.5.3. Corrélation des réponses de asset à différents chocs externes

\begin{tabular}{|c|c|c|c|}
\hline & wprices & usintrte & emei \\
\hline AG-BO & 0,57 & $-0,37$ & $-0,68$ \\
\hline AG-BR & 0,41 & $-0,19$ & 0,72 \\
\hline AG-CL & 0,58 & 0,93 & $-0,58$ \\
\hline AG-PY & $-0,94$ & $-0,41$ & $-0,46$ \\
\hline AG-UY & 0,56 & $-0,72$ & 0,99 \\
\hline BO-BR & 0,93 & 0,98 & $-0,34$ \\
\hline BO-CL & 0,85 & $-0,34$ & 0,63 \\
\hline BO-PY & $-0,75$ & 0,99 & 0,31 \\
\hline BO-UY & 0,84 & 0,91 & $-0,73$ \\
\hline BR-CL & 0,88 & $-0,18$ & 0,15 \\
\hline BR-PY & $-0,63$ & 0,97 & $-0,92$ \\
\hline BR-UY & 0,82 & 0,82 & 0,74 \\
\hline CL-PY & $-0,69$ & $-0,36$ & $-0,40$ \\
\hline CL-UY & 0,61 & $-0,68$ & $-0,55$ \\
\hline PY-UY & $-0,74$ & 0,92 & $-0,51$ \\
\hline
\end{tabular}

Tableau A.5.4. Corrélation des réponses de $m$ à différents chocs externes

\begin{tabular}{|c|c|c|c|}
\hline & wprices & usintrte & emei \\
\hline AG-BO & 0,64 & $-0,94$ & 0,66 \\
\hline AG-BR & $-0,81$ & $-0,61$ & 0,94 \\
\hline AG-CL & $-0,15$ & $-0,78$ & 0,97 \\
\hline AG-PY & $-0,86$ & $-0,24$ & 0,76 \\
\hline AG-UY & 0,57 & $-0,95$ & 0,80 \\
\hline BO-BR & $-0,82$ & 0,43 & 0,86 \\
\hline BO-CL & 0,27 & 0,63 & 0,79 \\
\hline BO-PY & $-0,73$ & $-0,01$ & 0,96 \\
\hline BO-UY & 0,81 & 0,95 & 0,81 \\
\hline BR-CL & 0,27 & 0,86 & 0,98 \\
\hline BR-PY & 0,98 & 0,88 & 0,94 \\
\hline BR-UY & $-0,58$ & 0,44 & 0,92 \\
\hline CL-PY & 0,38 & 0,68 & 0,88 \\
\hline CL-UY & 0,56 & 0,69 & 0,89 \\
\hline PY-UY & $-0,48$ & 0,07 & 0,94 \\
\hline
\end{tabular}


Tableau A.5.5. Corrélation des réponses de ninrte à différents chocs externes

\begin{tabular}{|c|c|c|c|}
\hline & wprices & Usintrte & Emei \\
\hline AG-BO & $-0,29$ & 0,83 & 0,95 \\
\hline AG-BR & $-0,11$ & $-0,43$ & 0,62 \\
\hline AG-CL & 0,09 & $-0,37$ & 0,97 \\
\hline AG-PY & 0,17 & 0,67 & $-0,95$ \\
\hline AG-UY & 0,51 & $-0,41$ & $-0,96$ \\
\hline BO-BR & 0,67 & 0,14 & 0,81 \\
\hline BO-CL & 0,61 & $-0,73$ & 0,93 \\
\hline BO-PY & 0,58 & 0,96 & $-0,98$ \\
\hline BO-UY & $-0,53$ & $-0,83$ & $-0,99$ \\
\hline BR-CL & 0,98 & $-0,51$ & 0,56 \\
\hline BR-PY & 0,95 & 0,37 & $-0,75$ \\
\hline CL-PY & $-0,58$ & $-0,60$ & $-0,78$ \\
\hline CL-UY & 0,99 & $-0,84$ & $-0,96$ \\
\hline PY-UY & $-0,46$ & 0,75 & $-0,95$ \\
\hline
\end{tabular}

\section{RÉFÉRENCES}

Bayoumi, T. et Eichengreen, B., 1993. Is There a Conflict Between EC Enlargement and European Monetary Unification, Greek Economic Review, 15(1), 131-154.

Bordo, M.D. et Murshid, A.P., 2002. Globalization and Changing Patterns in the International Transmission of Shocks in Financial Markets, NBER Working Paper 9019.

Calvo, G., Leiderman, L. et Reinhart, C., 1994. "The Capital Inflows Problem: Concepts and Issues", Contemporary Economic Policy, 12(3), 54-66.

Calvo, G. et Mendoza, E., 1998. Rational Herd Behaviour and the Globalization of Securities Markets, mimeo, University of Maryland.

Canova, F., 2003. The Transmission of US Shocks to Latin America, CEPR Discussion Paper Series, 3963.

Chang, R. et Velasco, A., 2000. Banks, Debt Maturity and Financial Crises, Journal of Economics Theory, 51, 169-194.

Clarida, R. et Gali, J., 1994. Sources of Real Exchange Rate Fluctuations: How Important are Nominal Shocks?, NBER Working Papers 4658.

Corsetti, G., Pesenti, P. et Roubini, N., 1999. Paper Tigers? A Model of the Asian Crisis. European Economic Review, 43(7), 1211-1236.

Cushman, D.O. et Zha, T., 1997. Identifying Monetary Policy in Small open Economy Under Flexible Exchange Rate, Journal of Monetary Economics, 39, 433-448.

Gali, J., 1992. Does the IS-LM Model Fit US Postwar Data ?, Quarterly Journal of Economics, CVII, 708-738.

Kaminsky, G.L., Reinhart, C.M. et Vegh, C.A., 2003. The Unholy Trinity of Financial Contagion, Journal of Economic Perspectives, American Economic Association, 17(4), 51-74.

Kim, S. et Roubini, N., 2000. Exchange Rate Anomalies in Industrialized Countries: A Solution with a Structural VAR Approach, Journal of Monetary Economics, 45, 561586.

Lack, C. et Lenz, C., 2000. A Program for the Identification of Structural Var Models, WWZ-Studie, 58, april. 
Mackowiak, B., 2006. External Shocks, US Monetary Policy and Macroeconomic Fluctuations in Emerging Markets, SFB 649 Discussion Papers.

Runkle, D., 1987.Vector Autoregressions and Reality, Journal of Business and Economic Statistics, 5, 435-442.

Sims, C.A., 1992. Interpreting the Macroeconomic Time Series Facts: The Effects of Monetary Policy. European Economic Review, 36,975-1000.

Sims, C.A. et Zha, T., 1999. Error Bands for Impulse Responses, Econometrica, 67(5), 1113-1155. 